\title{
Coulomb explosion imaging of small polyatomic molecules with ultrashort $x$-ray pulses
}

\author{
X. Li ${ }^{1,2,},{ }^{*}$ A. Rudenko, ${ }^{1, \dagger}$ M. S. Schöffler, ${ }^{3}$ N. Anders, ${ }^{3}$ Th. M. Baumann, ${ }^{4}$ S. Eckart, ${ }^{3}$ B. Erk, ${ }^{5}$ A. De Fanis, ${ }^{4}$ K. Fehre,${ }^{3}$ \\ R. Dörner, ${ }^{3}$ L. Foucar, ${ }^{6}$ S. Grundmann, ${ }^{3}$ P. Grychtol, ${ }^{4}$ A. Hartung, ${ }^{3}$ M. Hofmann, ${ }^{3}$ M. Ilchen,,${ }^{4}$ Ch. Janke, ${ }^{3}$ G. Kastirke, ${ }^{3}$ \\ M. Kircher, ${ }^{3}$ K. Kubicek, ${ }^{4,8}$ M. Kunitski,${ }^{3}$ T. Mazza, ${ }^{4}$ S. Meister, ${ }^{9}$ N. Melzer, ${ }^{3}$ J. Montano, ${ }^{4}$ V. Music, ${ }^{4,7}$ G. Nalin, ${ }^{3}$ \\ Y. Ovcharenko, ${ }_{4}^{4}$ Ch. Passow, ${ }^{4}$ A. Pier, ${ }^{3}$ N. Rennhack, ${ }^{4}$ J. Rist,${ }^{3}$ D. E. Rivas, ${ }^{4}$ I. Schlichting, ${ }^{6}$ L. Ph. H. Schmidt ${ }^{3}$ \\ Ph. Schmidt, ${ }^{4,7}$ J. Siebert, ${ }^{3}$ N. Strenger, ${ }^{3}$ D. Trabert, ${ }^{3}$ F. Trinter, ${ }^{3,5,10}$ I. Vela-Perez ${ }^{3}$ R. Wagner, ${ }^{4}$ P. Walter, ${ }^{2}$ \\ M. Weller, ${ }^{3}$ P. Ziolkowski, ${ }^{4}$ A. Czasch,${ }^{3}$ D. Rolles, ${ }^{1}$ M. Meyer, ${ }^{4}$ T. Jahnke, ${ }^{4}$ and R. Boll $\oplus^{4, 末}$ \\ ${ }^{1}$ J.R. Macdonald Laboratory, Department of Physics, Kansas State University, Manhattan, Kansas 66506, USA \\ ${ }^{2}$ Linac Coherent Light Source, SLAC National Accelerator Laboratory, Menlo Park, California 94025, USA \\ ${ }^{3}$ Institut für Kernphysik, Goethe-Universität, Max-von-Laue-Strasse 1, 60438 Frankfurt am Main, Germany \\ ${ }^{4}$ European XFEL, Holzkoppel 4, 22869 Schenefeld, Germany \\ ${ }^{5}$ Deutsches Elektronen-Synchrotron (DESY), Notkestrasse 85, 22607 Hamburg, Germany \\ ${ }^{6}$ Max-Planck-Institut für Medizinische Forschung, 69120 Heidelberg, Germany \\ ${ }^{7}$ Institut für Physik und CINSaT, Universität Kassel, Heinrich-Plett-Strasse 40, 34132 Kassel, Germany \\ ${ }^{8}$ The Hamburg Centre for Ultrafast Imaging, 22761 Hamburg, Germany \\ ${ }^{9}$ Max-Planck-Institute for Nuclear Physics, 69117 Heidelberg, Germany \\ ${ }^{10}$ Molecular Physics, Fritz-Haber-Institut der Max-Planck-Gesellschaft, Faradayweg 4-6, 14195 Berlin, Germany
}

(Received 18 January 2021; revised 22 September 2021; accepted 23 September 2021; published 13 January 2022)

\begin{abstract}
Ultrashort x-ray pulses from free-electron lasers can efficiently charge up and trigger the full fragmentation of molecules. By coincident detection of up to five ions resulting from rapid Coulomb explosion of highly charged iodomethane, we show that the full three-dimensional equilibrium geometry of this prototypical polyatomic system can be determined from the measured ion momenta with the help of a charge buildup model. Supported by simulations of how the ion momenta would reflect specific changes in molecular bond lengths and angles, we demonstrate that Coulomb-explosion imaging with ultrashort $\mathrm{x}$-ray pulses is a promising technique for recording movies of multidimensional nuclear wave packets, including hydrogen motions.
\end{abstract}

DOI: 10.1103/PhysRevResearch.4.013029

\section{INTRODUCTION}

Novel methods for gas-phase molecular imaging offer the capability of directly probing the position of each atom in an ultrafast reaction, thus pushing our "vision limits" towards sub-Ångström spatial resolution, and a response time of a few femtoseconds. The past decade has witnessed key steps in this direction with the implementation of several complementary imaging techniques based on photon or electron scattering. X-ray diffraction, conventionally applied for imaging crystallized structures, was demonstrated to be able to make snapshots of ensembles of laser-aligned molecules with intense pulses generated by $\mathrm{x}$-ray free-electron lasers (XFELs) [1]. Moreover, this technique was used to produce a "molecular movie" of a prototypical ring-opening reaction [2], as well as to image coherent vibrational wave packets in diatomic [3] and more complex [4] molecules. In parallel, diffraction with short electron pulses [5] was employed to reconstruct

\footnotetext{
*xiangli@slac.stanford.edu

†rudenko@phys.ksu.edu

†rebecca.boll@xfel.eu
}

Published by the American Physical Society under the terms of the Creative Commons Attribution 4.0 International license. Further distribution of this work must maintain attribution to the author(s) and the published article's title, journal citation, and DOI. equilibrium molecular geometries [6]. With a temporal resolution approaching 100 fs [7], it enabled direct visualization of vibrational motions [8], the dynamics in the vicinity of a conical intersection [9], and a ring-opening reaction [10]. To utilize electron diffraction with even finer (potentially sub-10-fs) temporal resolution, alternative techniques based on "self-imaging" of a molecule with its own photoelectrons [11-15] or Auger electrons [16] have been demonstrated.

A different technique, not based on photon or electron scattering, is Coulomb-explosion imaging (CEI). It was first demonstrated by sending swift ionic molecules through a thin solid film, which stripped off all valence electrons and resulted in rapid fragmentation [17]. The resulting ion momenta were recorded on a position-sensitive detector and contain information on the molecular structure. More recently, CEI was often realized through photoionization, induced by either intense laser pulses [18-22] or X-ray pulses [23-26]. One key motivation to use light pulses to trigger Coulomb explosion is to apply this technique as a probe in time-resolved measurements. However, the full molecular geometry could only be studied mainly for molecules with few atoms [19,22,27,28]. For larger systems, CEI experiments were typically restricted to addressing a specific geometry-related question, such as following signatures of hydrogen migration [20,21,23,29], or distinguishing between isomers [24,26] or enantiomers [30]. One major limitation for applying CEI to larger molecules is that a molecule is often only partially fragmented after the 
photoionization $[20,21,24,26]$. Nevertheless, even for cases where a full fragmentation was achieved, and all fragments could be detected in coincidence [23,30,31], the full quantitative three-dimensional (3D) molecular geometry was not revealed, mainly because of the complexity of the multiple ionization process and due to non-negligible geometry changes during the time required to reach the final state.

Intense femtosecond XFEL pulses can charge up molecules very efficiently via sequential multiphoton ionization [32-35]. Here, we demonstrate how the use of intense XFEL pulses, combined with a coincident ion momentum imaging setup and an appropriate data representation scheme, can reveal the complete 3D geometry of iodomethane $\left(\mathrm{CH}_{3} \mathrm{I}\right)$, a prototypical system for ultrafast photochemistry. With the help of a simple charge buildup model, we show that the retrieval of bond lengths and angles of the molecule can be achieved through an optimization approach, so long as the charge-up process is well characterized. Furthermore, by simulating how the ion momenta would reflect specific changes in molecular geometry, we demonstrate that CEI employing ultrashort XFEL pulses is a promising technique for recording "movies" of multidimensional nuclear wave packets.

\section{RESULTS}

The experiment was carried out using a COLTRIMS (cold target recoil ion momentum spectroscopy) reaction microscope $[36,37]$ that is part of the Small Quantum Systems (SQS) scientific instrument of the European XFEL [38]. $\mathrm{CH}_{3} \mathrm{I}$ molecules in a dilute gas jet were ionized by $\mathrm{x}$-ray pulses with a photon energy of $2 \mathrm{keV}$, a pulse duration of $\sim 25 \mathrm{fs}$ (estimated from the electron bunch length), a pulse energy of $0.8 \mathrm{~mJ}$, and a focal spot diameter of $\sim 1.4 \mu \mathrm{m}$ (full width at half maximum). After sequential multiphoton ionization, Auger decays and intramolecular charge redistribution [32-35], the molecule fragments into $\left[\mathrm{I}^{m+}, \mathrm{C}^{n+}, k \times \mathrm{H}^{+}\right]$.

The yield of carbon and iodine ions detected in coincidence is plotted in Fig. 1(a), where $m$ ranges from 1 to 30 and $n$ from 1 to 5. Figure 1(b) displays the average number $k$ of hydrogen ions for each of the fragmentation pairs $\left[\mathrm{I}^{m+}, \mathrm{C}^{n+}\right]$ with $m \leqslant 10$. It is calculated assuming momentum conservation: $-P_{\mathrm{I} x}=P_{\mathrm{C} x}+k \times P_{\mathrm{H} x}$, where $P_{\mathrm{I} x}, P_{\mathrm{C} x}$, and $P_{\mathrm{H} x}$ are the respective momenta of iodine, carbon, and hydrogen ions along the $x$ axis (parallel to the iodine ion emission direction). The number of hydrogen ions saturates at 3 for iodine charge states above $\mathrm{I}^{5+}$, implying a full explosion of the $\mathrm{CH}_{3} \mathrm{I}$ molecule.

For the CEI analysis, we choose the channel $\left[\mathrm{I}^{9+}, \mathrm{C}^{3+}\right.$, $3 \times \mathrm{H}^{+}$] as an example, but the same procedure can be applied to any full-fragmentation channel. Three other cases are displayed in Appendix C, Fig. 6. The events are identified by the coincident detection of at least two $\mathrm{H}^{+}$, together with $\mathrm{I}^{9+}$ and $\mathrm{C}^{3+}$. Figure 2 shows the 3D molecular-frame momentum distribution of the fragment ions. The iodine and carbon ions essentially show a back-to-back emission while the three distinct spatial maxima at lower absolute momenta can be visually assigned to the individual hydrogen ions. The assignment of the color coding in Fig. 2 was not done visually, but was achieved by applying the $k$-means clustering algorithm [39-41], in order to develop a generally applicable method for identifying the individual atoms automatically, suitable for molecules containing more than one atom of the same
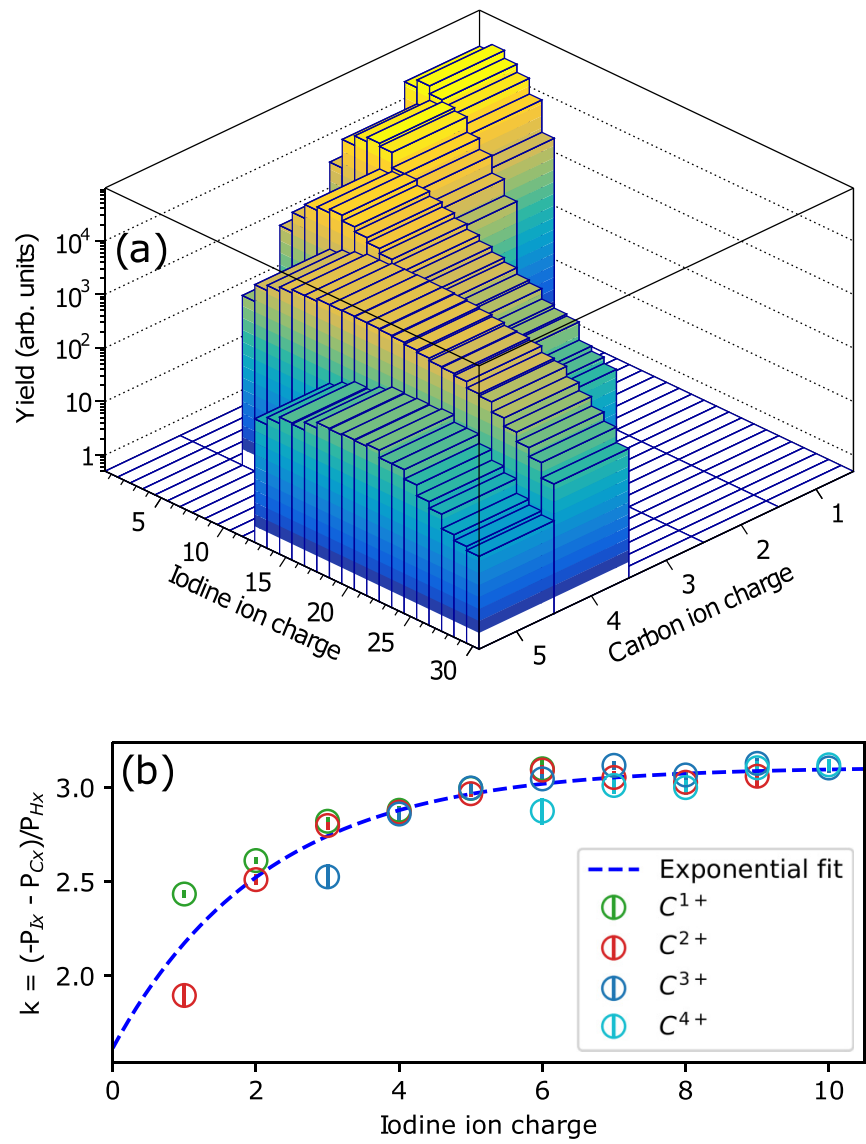

FIG. 1. Fragmentation of $\mathrm{CH}_{3} \mathrm{I}$ molecules after ionization by 2-keV, 0.8-mJ x-ray pulses. (a) Charge state distribution of carbon and iodine ions recorded in coincidence. (b) Average number $k$ of hydrogen ions produced from the fragmentation of a $\mathrm{CH}_{3} \mathrm{I}$ molecule for carbon and iodine ion pairs $\left[\mathrm{I}^{m+}, \mathrm{C}^{n+}\right]$ with $m \leqslant 10 . k$ is calculated by momentum conservation of the measured momenta (shown in Appendix C, Fig. 5), assuming that neutral $\mathrm{H}$ do not receive any momentum. The dashed line is an exponential fit to guide the eye.

type. When using the current data representation scheme, the atomic species are identified by their mass-over-charge ratio, and the number of atoms of each species is identified by the clustering algorithm. It is remarkable that under coincidence conditions and upon complete fragmentation of the molecules, no prior knowledge is required on the type and number of the atoms forming the molecule. This imaging technique is thus capable of identifying and imaging unknown molecules, and is suitable for imaging the full geometry of transients during ultrafast reactions. Although we have not tested its limitations, we expect our method to run into difficulties with molecules containing several tens of atoms, because the multiple coincidence detection for such large molecules can become prohibitive even with high-repetition-rate light sources.

The centroids of the ion momentum distributions in Fig. 2 after outlier removal (see Appendix B) are shown in Fig. 3 as spheres, with the steel-blue arrows indicating the momentum directions. The arrows for iodine and carbon are almost parallel to the $x$ axis. The hydrogen ion centroids are evenly distributed along the golden circle, and the angles between the projections of the three hydrogen ion arrows on the $y-z$ plane are $116^{\circ} \pm 8^{\circ}, 121^{\circ} \pm 9^{\circ}$, and $123^{\circ} \pm 7^{\circ}$, respectively. 


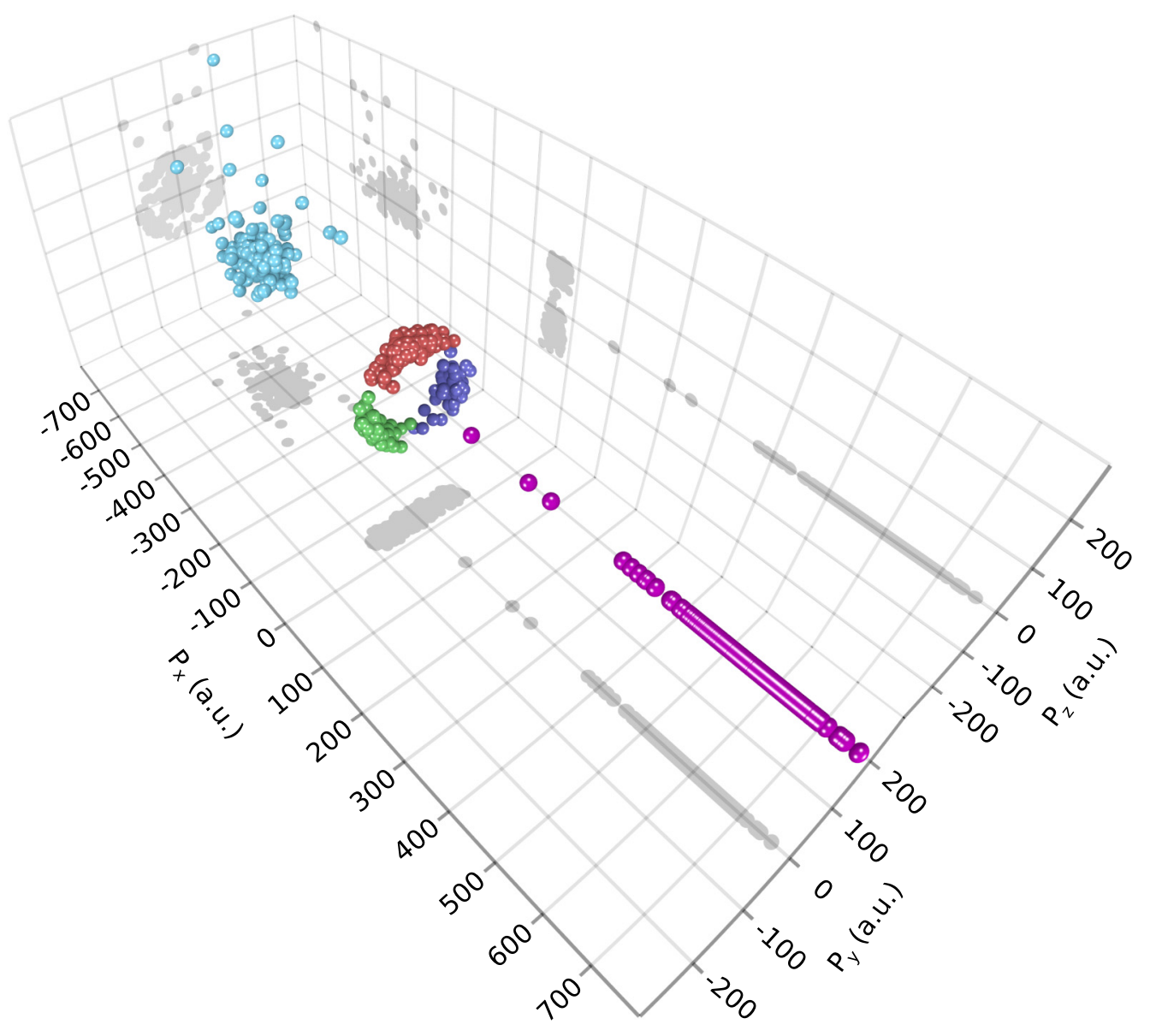

FIG. 2. Molecular-frame momentum distributions of the ions in the fragmentation channel $\left[\mathrm{I}^{9+}\right.$ (purple), $\mathrm{C}^{3+}$ (cyan), $3 \times \mathrm{H}^{+}$(red, green and blue)]. The coordinate frame is chosen such that the $\mathrm{I}^{9+}$ momentum lies along the $x$ axis, and the $z$ axis is defined such that the momentum sum of the carbon ion and the red hydrogen ion lies in the $x-z$ plane with $z>0$. Also shown are the projections on the three perpendicular planes.

Based on these measurements, the distribution of the three cluster centroids of the hydrogen ions can be regarded as having a cylindrical symmetry, in agreement with the known equilibrium geometry of iodomethane.

After having determined the cylindrical symmetry of the molecule, additional knowledge of the $\mathrm{I}-\mathrm{C}-\mathrm{H}$ bond angle and the $\mathrm{C}-\mathrm{I}$ and $\mathrm{C}-\mathrm{H}$ bond lengths is sufficient for a complete characterization of the molecular geometry. In order to obtain those, the data are compared to classical Coulomb-explosion simulations. In Fig. 3, the hollow tetrahedrons are the simulated momenta, assuming that the molecule is instantaneously charged up at the equilibrium geometry [42]. A reasonably good match is achieved, suggesting that the XFEL pulses charge up the molecules so efficiently that the process can be approximated as almost instantaneous. However, a close inspection of Fig. 3 reveals that the magnitudes of the simulated momenta are consistently larger than their experimental counterparts. This slight mismatch is due to the fact that the charge buildup process takes a finite time, during which the fragmentation already starts to set in, resulting in smaller fragment momenta. The charge evolution is determined by the competition between sequential ionization, deexcitation, and charge redistribution, taking place on the timescale of the XFEL pulse length $[35,43,44]$. As calculated by ab initio mod- eling with the Monte Carlo method [35,43,45,46], the average charge evolution can be modeled by the error function $\operatorname{Erf}(x)$. To incorporate this process into the Coulomb-explosion simulation, the time-dependent charge $Q_{X}(t)$ on an atom $X$ is simulated according to

$$
Q_{X}(t)=\left(Q_{X}^{f}-Q_{X}^{i}\right) \frac{\operatorname{Erf}\left(\frac{4 \sqrt{\ln (2)}}{\tau_{X}} t-\frac{5}{2}\right)+1}{2}+Q_{X}^{i},
$$

where $X=\mathrm{I}, \mathrm{C}$, or $\mathrm{H} ; Q_{X}^{f}$ is the final fragment charge measured in the experiment; $Q_{X}^{i}$ is the initial fragment charge, or the amount of charge which can be considered as instantaneously obtained; and $\tau_{X}$ is the constant quantifying the timescale over which the remaining charge $\left(Q_{X}^{f}-Q_{X}^{i}\right)$ is acquired. To find the values of $Q_{X}^{i}$ and $\tau_{X}$ which best describe the charge-up process, we minimize the metric $M\left(Q_{\mathrm{I}}^{i}, \tau_{\mathrm{I}}, Q_{\mathrm{C}}^{i}, \tau_{\mathrm{C}}, Q_{\mathrm{H}}^{i}, \tau_{\mathrm{H}}\right)$ using the differential evolution global optimization algorithm $[47,48]$, with the metric given by

$$
\begin{aligned}
M= & \left(P_{\mathrm{C} x}^{\mathrm{sim}}-P_{\mathrm{C} x}\right)^{2}+3 \times\left(P_{\mathrm{H} x}^{\mathrm{sim}}-P_{\mathrm{H} x}\right)^{2} \\
& +3 \times\left(P_{\mathrm{H} \perp}^{\mathrm{sim}}-P_{\mathrm{H} \perp}\right)^{2},
\end{aligned}
$$




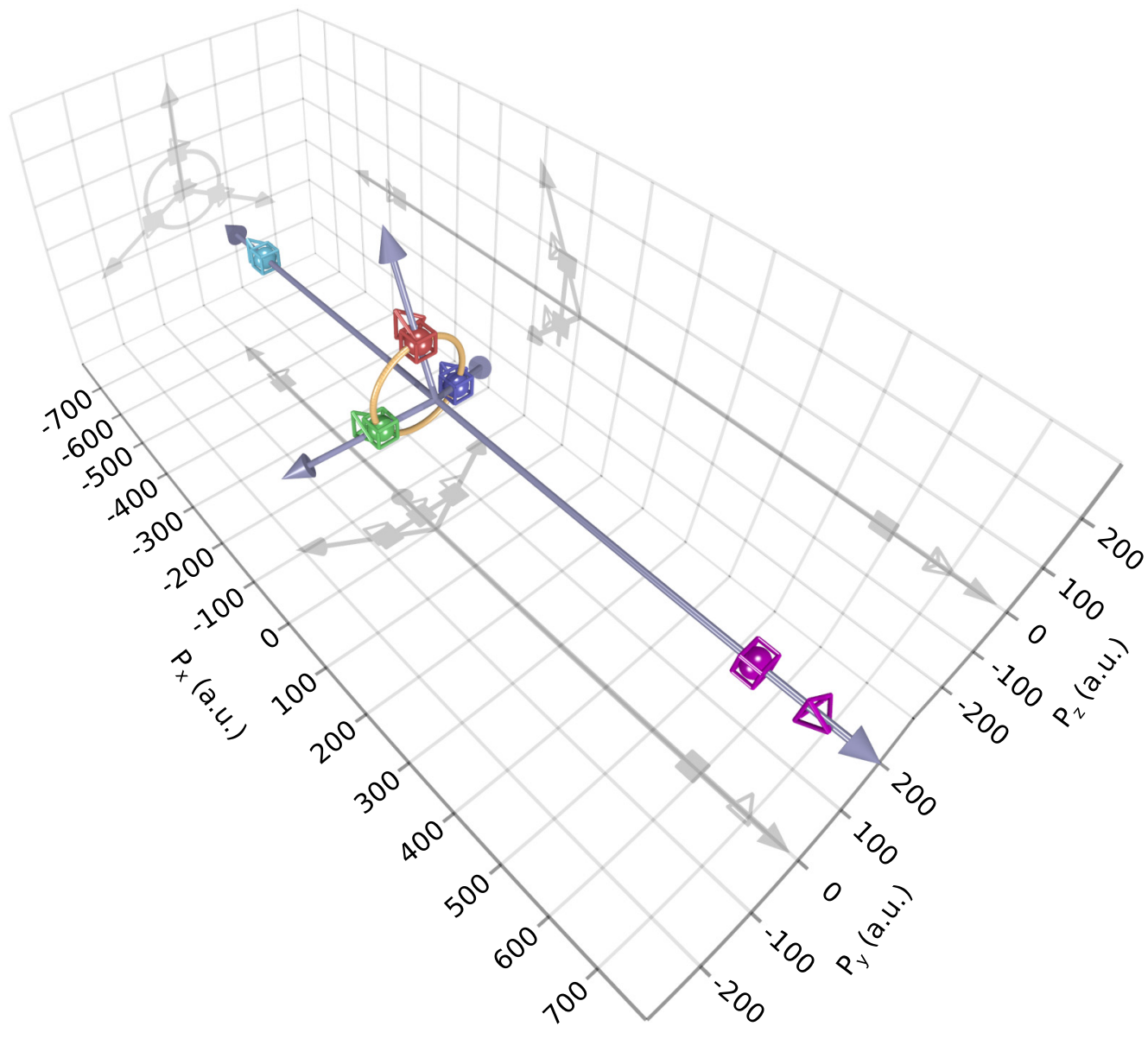

FIG. 3. Comparison between the experimental and simulated molecular-frame momenta of the ions in the fragmentation channel $\left[\mathrm{I}^{9+}\right.$ (purple), $\mathrm{C}^{3+}$ (cyan), $3 \times \mathrm{H}^{+}$(red, green and blue)]. The spheres are the centroids of the experimental distributions in Fig. 2 after outlier removal, with the three hydrogen spheres evenly distributed along the golden circle. The momentum directions are shown by the steel-blue arrows. Tetrahedrons and cubes are the simulated momenta based on the instantaneous charge-up model and the charge buildup model, respectively. The three hydrogen ion centroids are evenly distributed around the golden circle which is centered around and perpendicular to the $x$ axis. Also shown are the projections on the three perpendicular planes.

where $P_{\mathrm{C} x}$ and $P_{\mathrm{H} x}$ are the average carbon and hydrogen ion momenta along the $x$ axis, $P_{H \perp}$ is the average hydrogen ion momentum along the outward direction perpendicular to the $x$ axis, and the momenta with superscript "sim" are from the Coulomb-explosion simulation which depends on the parameters $Q_{X}^{i}$ and $\tau_{X}$ of the charge buildup model. The optimized parameters $Q_{X}^{i}$ and $\tau_{X}$ are summarized in Appendix $\mathrm{C}$, Table I, with the optimized charge-up time $\tau_{X}$ being $\sim 10$ fs. The corresponding simulated molecular-frame momenta are plotted as hollow cubes in Fig. 3, which overlap almost perfectly with the ones measured in the experiment.

If the charge buildup process is well characterized, it is possible to retrieve the molecular geometry from the measured momenta in Fig. 3. As a first attempt for such a retrieval, we use Eq. (1) with the optimized parameters $Q_{X}^{i}$ and $\tau_{X}$ for the charge buildup model. To retrieve the values of the $\mathrm{C}-\mathrm{I}$ and $\mathrm{C}-\mathrm{H}$ bond lengths $\left(l_{\mathrm{C}-\mathrm{I}}\right.$ and $\left.l_{\mathrm{C}-\mathrm{H}}\right)$ and the $\mathrm{I}-\mathrm{C}-\mathrm{H}$ angle $\left(\angle_{\mathrm{I}-\mathrm{C}-\mathrm{H}}\right)$, we take an optimization approach, with the same metric as in Eq. (2), which is now a function of $l_{\mathrm{C}-\mathrm{I}}$, $l_{\mathrm{C}-\mathrm{H}}$, and $\angle_{\mathrm{I}-\mathrm{C}-\mathrm{H}}$. When applying (very relaxed) boundary conditions of $0.1-10 \AA$ for the two bond lengths and $50^{\circ}-150^{\circ}$ for the angle, the optimization is found to converge to the expected values for the $\mathrm{CH}_{3} \mathrm{I}$ molecule at equilibrium [42], with $l_{\mathrm{C}-\mathrm{I}}=2.1 \pm 0.2 \AA, l_{\mathrm{C}-\mathrm{H}}=1.1 \pm 0.4 \AA$, and $\angle_{\mathrm{I}-\mathrm{C}-\mathrm{H}}=$ $107^{\circ} \pm 3^{\circ}$. In future studies, the charge buildup process by XFEL pulses could be characterized either by independent measurements or by $a b$ initio simulations, which can then be used in conjunction with the current optimization procedure to retrieve the molecular geometry.

\section{DISCUSSION}

Up to this point, we have demonstrated how CEI can be used to determine the 3D molecular geometry, including all bond lengths and angles. In the following, we demonstrate that multidimensional nuclear motions are clearly reflected in the calculated 3D molecular-frame ion momenta, suggesting that our approach can also be applied to direct spatiotemporal imaging of multidimensional nuclear motions in a pump-probe experiment. Although the example is on imaging molecular vibrations, the technique can similarly be applied to imaging other dynamics involving nuclear motions, such as those in roaming reactions [29]. 

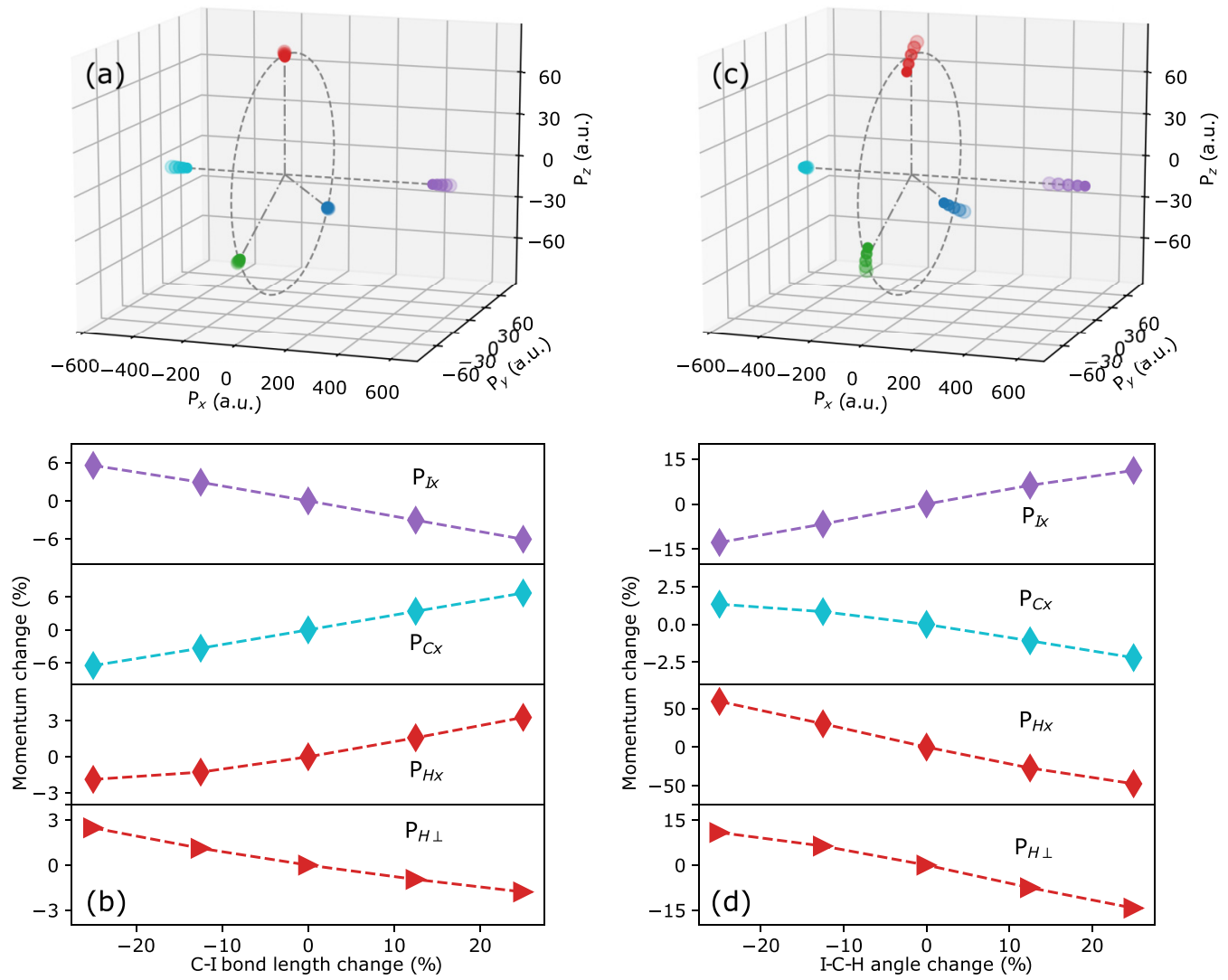

FIG. 4. Simulated change of molecular-frame momentum of the fragmentation channel $\left[\mathrm{I}^{9+}\right.$ (purple), $\mathrm{C}^{3+}$ (cyan), $3 \times \mathrm{H}^{+}$(red, green, and blue)] as a function of the changes in the $\mathrm{C}-\mathrm{I}$ bond length and the $\mathrm{I}-\mathrm{C}-\mathrm{H}$ angle. (a) Simulated molecular-frame momentum for five different initial C-I bond lengths changed relative to the equilibrium value $\mathrm{l}_{\mathrm{C}-\mathrm{I}}^{0}$ by $\pm 25 \%$ in steps of $12.5 \%$ [C-I "stretch" mode $\left.\left(v_{3}\right)\right]$. The smaller the percentage change, the larger and more transparent are the data symbols. (b) Percentage change of the fragment average momentum as a function of the $\mathrm{C}-\mathrm{I}$ bond length percentage change, relative to the equilibrium. (c) Simulated molecular-frame momentum for five different initial I-C-H angles changed relative to the equilibrium value $\angle_{\mathrm{I}-\mathrm{C}-\mathrm{H}}^{0}$ by $\pm 25 \%$ in steps of $12.5 \%\left[\mathrm{CH}_{3}\right.$ "umbrella" mode $\left.\left(v_{2}\right)\right]$. The larger the percentage change, the larger and more transparent are the data symbols. (d) Percentage change of the fragment average momentum as a function of the $\mathrm{I}-\mathrm{C}-\mathrm{H}$ angle percentage change, relative to the equilibrium.

In $\mathrm{CH}_{3} \mathrm{I}$ molecules, vibrational wave-packet dynamics along the C-I "stretch" $\left(v_{3}\right)[49,50]$ and the $\mathrm{CH}_{3}$ "umbrella" $\left(v_{2}\right)$ modes [49] have been observed after infrared excitation. We investigate how the molecular-frame momentum changes when the $\mathrm{C}-\mathrm{I}$ bond length or the $\mathrm{I}-\mathrm{C}-\mathrm{H}$ angle are varied $\pm 25 \%$ in steps of $12.5 \%$. For each geometry, a simulation procedure similar to the one used to produce the calculations in Fig. 3 is carried out. Since the C-I stretch and the $\mathrm{CH}_{3}$ umbrella modes correspond to only small displacements of each atom from the equilibrium geometry and the charge buildup process is not expected to vary dramatically due to such motions, the time constant $\tau_{X}$ for the vibration simulation is assumed to be identical to that of the equilibrium geometry. The respective centroids of the five fragment clusters are calculated and plotted in Figs. 4(a) and 4(c) for the stretch and umbrella motions, respectively. The trajectory traced out by the iodine and carbon ion centroids in Fig. 4(a) reflects the C-I stretch motion, whereas the change of the hydrogen ion centroids in Fig. 4(c) reflects the $\mathrm{CH}_{3}$ umbrella motion. The relative changes in the momentum of the different ion fragments are plotted in Figs. 4(b) and 4(d). They show that nuclear vibrations along different modes induce different kinds of changes, of up to several tens of atomic units in the fragment ion momenta, which can be well resolved by the COLTRIMS reaction microscope. Such signature changes in momentum space can, therefore, be used to decompose the overall nuclear dynamics observed by CEI into different vibrational components.

Our work shows that Coulomb-explosion imaging using ultrashort, intense XFEL pulses in combination with a 3D data representation in momentum space is a promising technique for imaging the structures of polyatomic molecules as well as multidimensional nuclear wave-packet dynamics. With future upgrades on XFEL repetition rate, the time required to take a "molecular snapshot" like Fig. 3 has the potential to be reduced to seconds, thus shortening the total acquisition time needed for making "molecular movies" and therefore dramatically increasing our capability of looking into the molecular world.

Data recorded for the experiment at the European XFEL are available at [51]. Requests for materials should be addressed to the authors. 


\section{ACKNOWLEDGMENTS}

This work is supported by the U.S. Department of Energy, Office of Science, Basic Energy Sciences, Chemical Sciences, Geosciences, and Biosciences Division, who supported X.L. under Grant No. DE-SC0019451, and A.R. and D.R. under Grant No. DE-FG02-86ER13491. We acknowledge European XFEL in Schenefeld, Germany, for provision of x-ray freeelectron laser beam time at the SQS instrument and would like to thank the staff for their assistance. R.D., M.S.S., K.F., T.J., and M.H. acknowledge support from the Deutsche Forschungsgemeinschaft via Sonderforschungsbereich 1319 (ELCH). M.I., V.M., and Ph.S. acknowledge funding from the Volkswagen Foundation within a Peter Paul Ewald Fellowship. K.K. acknowledges support by the Cluster of Excellence Advanced Imaging of Matter of the Deutsche Forschungsgemeinschaft (DFG), EXC 2056, Project ID 390715994.

\section{APPENDIX A: EXPERIMENT AND DATA ANALYSIS}

The experiment was performed at the Small Quantum Systems (SQS) instrument of the European XFEL. The x-ray pulses with 25-fs duration (estimated from the electron bunch length), $2-\mathrm{keV}$ photon energy, and $0.8-\mathrm{mJ}$ pulse energy were focused by a pair of Kirkpatrick-Baez mirrors to the center of the COLTRIMS reaction microscope [36,37] which is available as a user endstation at SQS, where it interacted with the $\mathrm{CH}_{3} \mathrm{I}$ molecule delivered by a supersonic gas jet. The ion fragments from this interaction were then guided by a homogeneous electric field to a time- and position-sensitive 120-mm microchannel plate equipped with a delay-line anode (RoentDek HEX100). The wave forms from the detector are amplified, digitized, and saved to disk. The signal arrival times are identified from the saved wave forms by a software "constant fraction discriminator." From the identified times, the position $(x, y)$ and the time $t$ of each detector hit are reconstructed. With the hit information, spectrometer geometry, and voltage settings, the ion fragment momentum acquired from the interaction is calculated with Newton's equations. In order to calibrate the experimental setup, a short run was taken with $\mathrm{N}_{2}$ molecules under the same experimental conditions. The calibration was completed by matching the distribution of the kinetic energy release from the $\mathrm{N}^{+} / \mathrm{N}^{+}$breakup channel with the published $\mathrm{N}_{2}$ data $[52,53]$. From these laboratory-frame momenta, the molecular-frame momenta are obtained and displayed in the form of Fig. 2. To be suitable for the retrieval of the molecular structure, the coincidence channel must satisfy two conditions. One is that it should be a full-fragmentation channel for a full reconstruction of the molecular geometry. Figure 1(b) shows the number of ejected protons for each $\left[\mathrm{I}^{m+}, \mathrm{C}^{n+}\right]$ ion pair, which saturates at 3 for iodine charge states above $5+$. Therefore, we regard all channels with iodine charge states of $5+$ and higher as fully fragmented. The second condition is that the amount of events per channel needs to be abundant for obtaining a meaningful result and the yield decreases with increasing charge state above $\mathrm{I}^{4+}$ as shown in Fig. 1(a). We found that, in the present dataset, this limited the retrieval to the channels with iodine ion charge states up to $10+$. Combining these two conditions, the channels with iodine charge states between $6+$ and $10+$ can, there- fore, be selected for retrieval. Apart from $\left[\mathrm{I}^{9+}, \mathrm{C}^{3+}, 3 \times \mathrm{H}^{+}\right]$ discussed in the main text, three more channels, $\left[\mathrm{I}^{6+}, \mathrm{C}^{2+}\right.$, $\left.3 \times \mathrm{H}^{+}\right],\left[\mathrm{I}^{7+}, \mathrm{C}^{3+}, 3 \times \mathrm{H}^{+}\right]$, and $\left[\mathrm{I}^{10+}, \mathrm{C}^{3+}, 3 \times \mathrm{H}^{+}\right]$, which fulfill these conditions, are shown in Fig. 6 of Appendix $\mathrm{C}$.

\section{APPENDIX B: 3D MOLECULAR-FRAME MOMENTUM DISTRIBUTION, THE RELATED ALGORITHMS, AND SIMULATIONS}

For each event with the selected fragmentation pattern, three $\left(\mathrm{I}^{9+}, \mathrm{C}^{3+}\right.$, and $\mathrm{H}^{+}$for Fig. 2) of the ions are used for fixing the molecular frame, and then the remaining arbitrary number of ions detected in the same event are plotted in that frame. Since the detection efficiency for each ion in a reaction microscope is less than 1 [54] and detecting all five ions in coincidence is unlikely, the current way of data representation, together with the efficient full fragmentation by XFEL pulses, is the key to be able to produce momentum plots such as Fig. 2, with the required total number of shots decreased by 3 to 4 orders of magnitude. In addition, no normalization with respect to the iodine ion momentum is applied. This makes the current representation more sensitive to reflect changes in the molecular geometry. The outlier removal from the raw molecular-frame momentum distribution was completed via the "elliptic-envelope" algorithm [55]. It fits a robust covariance estimate (ellipse) to the data and identifies the data points far away from the fit shape as outliers. The $k$-means algorithm
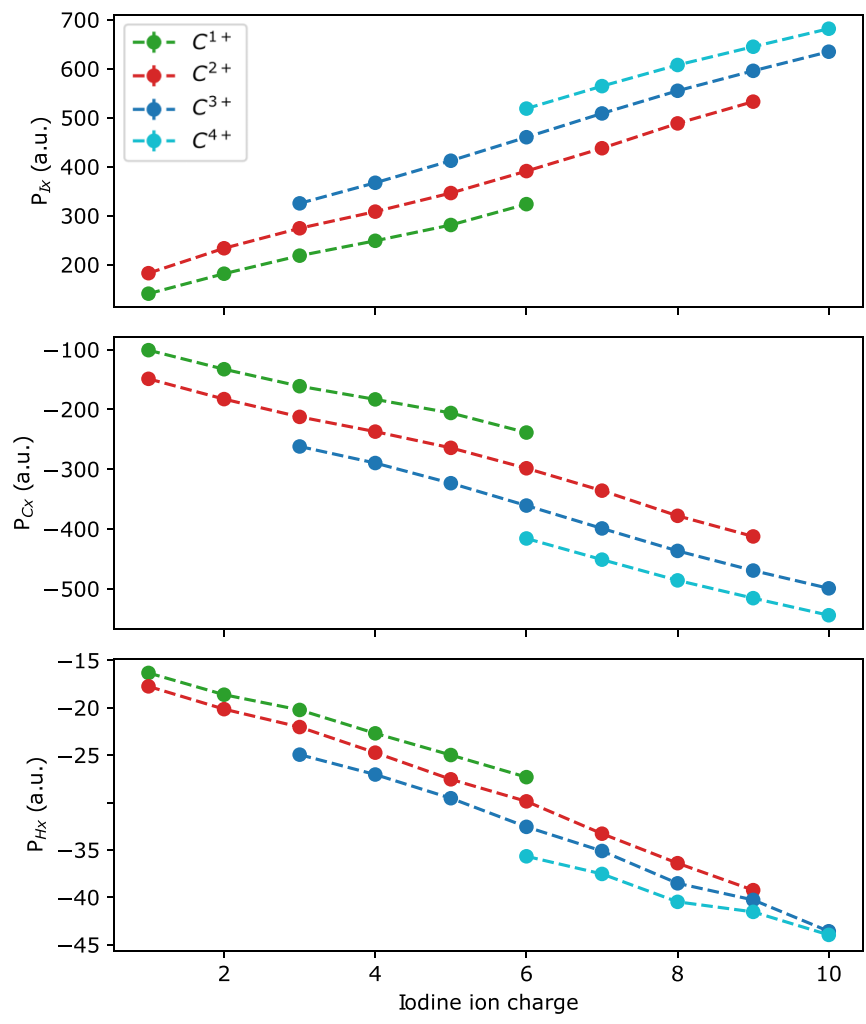

FIG. 5. Average momenta along the iodine ion momentum direction ( $x$ axis) of iodine (top), carbon (middle), and hydrogen (bottom) ions in different fragmentation channels, which are used to calculate the number of hydrogen ions in the fragmentation as shown in Fig. 1(b). 

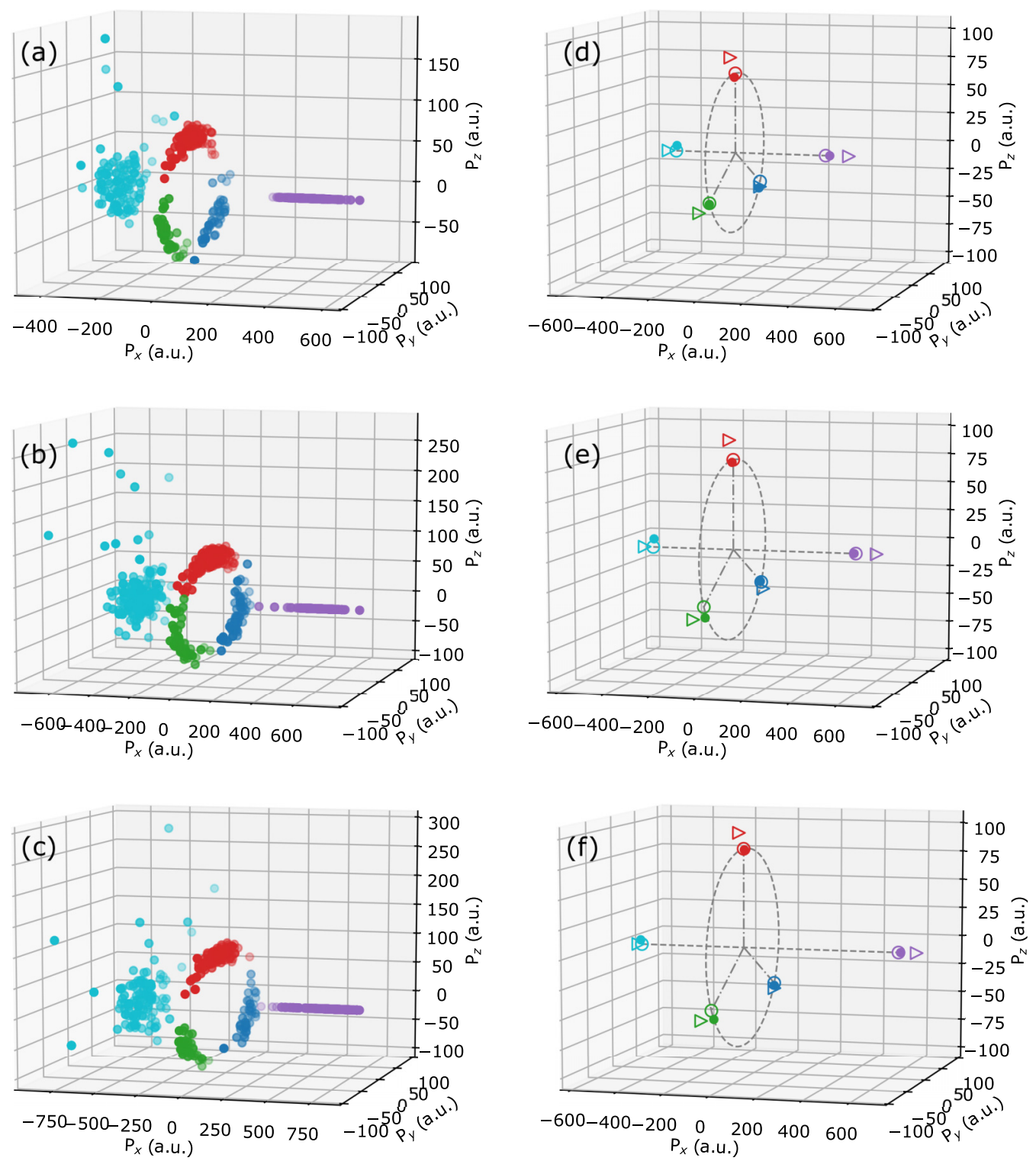

FIG. 6. Molecular-frame momentum distributions and the corresponding centroids of the ions in three different fragmentation channels: $\left[\mathrm{I}^{6+}, \mathrm{C}^{2+}, 3 \times \mathrm{H}^{+}\right]$[panels (a) and (d)], $\left[\mathrm{I}^{7+}, \mathrm{C}^{3+}, 3 \times \mathrm{H}^{+}\right]$[panels (b) and (e)], and $\left[\mathrm{I}^{10+}, \mathrm{C}^{3+}, 3 \times \mathrm{H}^{+}\right]$[panels (c) and (f)]. The same procedures as the ones to produce Fig. 2 are applied for plotting the momentum distributions (a), (b), and (c) on the left column. For the corresponding centroids plotted in panels (d), (e), and (f), circles are the experimental data. Triangles are the simulated momenta based on the instantaneous charge-up model. Open circles are the simulated momenta using the charge buildup model. The parameters of the model are obtained by optimization procedures explained in the main text and summarized in Table I.

$[39,40]$ used for grouping the $\mathrm{H}^{+}$ions aims to categorize the data points into $k$ clusters. It starts with $k$-cluster centroids from an initial guess (here, $k=3$ was chosen based on the existence of 3 maxima of the hydrogen ion momentum distribution in Fig. 2), assigns each data point to a cluster whose centroid is the closest to that data point, recalculates the updated cluster centroids, and repeats the assignment and recalculation steps until convergence is achieved. The Coulomb explosion of the molecule is simulated with the help of the eighth-order Runge-Kutta method. For the instantaneous charge-up model, the explosion starts at the initial molecular geometry when the fragments have already gained their final charges. For the charge buildup model, the fragment charge is updated at each integration step according to Eq. (1). During the differential evolution optimization, the metric evaluation for each iteration requires a complete Coulomb-explosion simulation with the temporary model parameters of that optimization step. The simulation does not include the relative momentum of the atoms prior to the arrival of the probe pulse, because the momentum due to the Coulomb explosion induced by the probe pulse is the dominant contribution. For example, during the vibrational motion in the $\tilde{A}$ state of $\mathrm{CH}_{3} \mathrm{I}^{+}$, the $\mathrm{C}-\mathrm{I}$ bond distance changes on the scale of 1 a.u. with a period of 113 fs [50], which corresponds to an initial momentum value of $\sim 2$ a.u. $(\sim 0.3 \%$ of the magnitude of the carbon or iodine ion momentum gained from Coulomb explosion). Since the value of the initial momentum is close to the momentum resolution of our measurement ( $\sim 3$ a.u.), 
TABLE I. The optimized charge buildup model parameters $Q_{X}^{i}$ and $\tau_{X}$ for four different fragmentation channels: $\left[\mathrm{I}^{6+}, \mathrm{C}^{2+}, 3 \times \mathrm{H}^{+}\right]$, $\left[\mathrm{I}^{7+}, \mathrm{C}^{3+}, 3 \times \mathrm{H}^{+}\right],\left[\mathrm{I}^{9+}, \mathrm{C}^{3+}, 3 \times \mathrm{H}^{+}\right]$, and $\left[\mathrm{I}^{10+}, \mathrm{C}^{3+}, 3 \times \mathrm{H}^{+}\right]$. The optimizations are performed with the differential evolution algorithm $[47,48]$.

\begin{tabular}{lccr}
\hline \hline Fragmentation channel & Ion fragment & $Q_{X}^{i}$ (a.u.) & $\tau_{X}(\mathrm{fs})$ \\
\hline$\left[\mathrm{I}^{6+}, \mathrm{C}^{2+}, 3 \times \mathrm{H}^{+}\right]$ & $\mathrm{I}^{6+}$ & 1.80 & 29.17 \\
& $\mathrm{C}^{2+}$ & 1.27 & 14.22 \\
& $\mathrm{H}^{+}$ & 0.78 & 1.72 \\
{$\left[\mathrm{I}^{7+}, \mathrm{C}^{3+}, 3 \times \mathrm{H}^{+}\right]$} & $\mathrm{I}^{7+}$ & 2.71 & 16.77 \\
& $\mathrm{C}^{3+}$ & 1.63 & 10.90 \\
& $\mathrm{H}^{+}$ & 0.15 & 5.10 \\
{$\left[\mathrm{I}^{9+}, \mathrm{C}^{3+}, 3 \times \mathrm{H}^{+}\right]$} & $\mathrm{I}^{9+}$ & 2.53 & 11.93 \\
& $\mathrm{C}^{3+}$ & 2.19 & 10.46 \\
{$\left[\mathrm{I}^{10+}, \mathrm{C}^{3+}, 3 \times \mathrm{H}^{+}\right]$} & $\mathrm{H}^{+}$ & 0.47 & 3.40 \\
& $\mathrm{I}^{10+}$ & 2.30 & 12.20 \\
& $\mathrm{C}^{3+}$ & 0.25 & 6.13 \\
& $\mathrm{H}^{+}$ & 0.31 & 2.72 \\
\hline \hline
\end{tabular}

neglecting this contribution only increases the error in the retrieval by approximately a factor of $\sim 1.2$ based on the above estimate.

\section{APPENDIX C: ION FRAGMENT MOMENTUM IN DIFFERENT FRAGMENTATION CHANNELS AND OPTIMIZED PARAMETERS OF THE CHARGE BUILDUP MODEL}

Figure 5 displays the average momenta in the molecular frame for different fragmentation channels, which are used to calculate the average number of hydrogen ions shown in Fig. 1(b). The molecular-frame momenta of the ions in three more fragmentation channels: $\left[\mathrm{I}^{6+}, \mathrm{C}^{2+}, 3 \times \mathrm{H}^{+}\right],\left[\mathrm{I}^{7+}, \mathrm{C}^{3+}\right.$, $\left.3 \times \mathrm{H}^{+}\right]$, and $\left[\mathrm{I}^{10+}, \mathrm{C}^{3+}, 3 \times \mathrm{H}^{+}\right]$are plotted in Fig. 6. The parameters of the charge buildup model optimized by the differential evolution algorithm for the three fragmentation channels as well as $\left[\mathrm{I}^{9+}, \mathrm{C}^{3+}, 3 \times \mathrm{H}^{+}\right]$are summarized in Table I.
[1] J. Küpper et al., X-Ray Diffraction from Isolated and Strongly Aligned Gas-Phase Molecules with a Free-Electron Laser, Phys. Rev. Lett. 112, 083002 (2014).

[2] M. P. Minitti, J. M. Budarz, A. Kirrander, J. S. Robinson, D. Ratner, T. J. Lane, D. Zhu, J. M. Glownia, M. Kozina, H. T. Lemke, M. Sikorski, Y. Feng, S. Nelson, K. Saita, B. Stankus, T. Northey, J. B. Hastings, and P. M. Weber, Imaging Molecular Motion: Femtosecond X-ray Scattering of an Electrocyclic Chemical Reaction, Phys. Rev. Lett. 114, 255501 (2015).

[3] J. M. Glownia, A. Natan, J. P. Cryan, R. Hartsock, M. Kozina, M. P. Minitti, S. Nelson, J. Robinson, T. Sato, T. van Driel, G. Welch, C. Weninger, D. Zhu, and P. H. Bucksbaum, SelfReferenced Coherent Diffraction X-Ray Movie of Ångstromand Femtosecond-Scale Atomic Motion, Phys. Rev. Lett. 117, 153003 (2016).

[4] B. Stankus, H. Yong, N. Zotev, J. M. Ruddock, D. Bellshaw, T. J. Lane, M. Liang, S. Boutet, S. Carbajo, J. S. Robinson, W. Du, N. Goff, Y. Chang, J. E. Koglin, M. P. Minitti, A. Kirrander, and P. M. Weber, Ultrafast X-ray scattering reveals vibrational coherence following Rydberg excitation, Nat. Chem. 11, 716 (2019).

[5] H. Ihee, V. A. Lobastov, U. M. Gomez, B. M. Goodson, R. Srinivasan, C.-Y. Ruan, and A. H. Zewail, Direct imaging of transient molecular structures with ultrafast diffraction, Science 291, 458 (2001).

[6] C. J. Hensley, J. Yang, and M. Centurion, Imaging of Isolated Molecules with Ultrafast Electron Pulses, Phys. Rev. Lett. 109, 133202 (2012).

[7] S. P. Weathersby, G. Brown, M. Centurion, T. F. Chase, R. Coffee, J. Corbett, J. P. Eichner, J. C. Frisch, A. R. Fry, M. Gühr, N. Hartmann, C. Hast, R. Hettel, R. K. Jobe, E. N. Jongewaard, J. R. Lewandowski, R. K. Li, A. M. Lindenberg, I. Makasyuk, J. E. May et al., Mega-electron-volt ultrafast electron diffraction at SLAC National Accelerator Laboratory, Rev. Sci. Instrum. 86, 073702 (2015).
[8] J. Yang, M. Guehr, X. Shen, R. Li, T. Vecchione, R. Coffee, J. Corbett, A. Fry, N. Hartmann, C. Hast, K. Hegazy, K. Jobe, I. Makasyuk, J. Robinson, M. S. Robinson, S. Vetter, S. Weathersby, C. Yoneda, X. Wang, and M. Centurion, Diffractive Imaging of Coherent Nuclear Motion in Isolated Molecules, Phys. Rev. Lett. 117, 153002 (2016).

[9] J. Yang, X. Zhu, T. J. A. Wolf, Z. Li, J. P. F. Nunes, R. Coffee, J. P. Cryan, M. Gühr, K. Hegazy, T. F. Heinz, K. Jobe, R. Li, X. Shen, T. Veccione, S. Weathersby, K. J. Wilkin, C. Yoneda, Q. Zheng, T. J. Martinez, M. Centurion et al., Imaging $\mathrm{CF}_{3} \mathrm{I}$ conical intersection and photodissociation dynamics with ultrafast electron diffraction, Science 361, 64 (2018).

[10] T. J. A. Wolf, D. M. Sanchez, J. Yang, R. M. Parrish, J. P. F. Nunes, M. Centurion, R. Coffee, J. P. Cryan, M. Gühr, K. Hegazy, A. Kirrander, R. K. Li, J. Ruddock, X. Shen, T. Vecchione, S. P. Weathersby, P. M. Weber, K. Wilkin, H. Yong, Q. Zheng et al., The photochemical ring-opening of 1,3cyclohexadiene imaged by ultrafast electron diffraction, Nat. Chem. 11, 504 (2019).

[11] C. I. Blaga, J. Xu, A. D. DiChiara, E. Sistrunk, K. Zhang, P. Agostini, T. A. Miller, L. F. DiMauro, and C. D. Lin, Imaging ultrafast molecular dynamics with laser-induced electron diffraction, Nature (London) 483, 194 (2012).

[12] R. Boll, D. Anielski, C. Bostedt, J. D. Bozek, L. Christensen, R. Coffee, S. De, P. Decleva, S. W. Epp, B. Erk, L. Foucar, F. Krasniqi, J. Küpper, A. Rouzée, B. Rudek, A. Rudenko, S. Schorb, H. Stapelfeldt, M. Stener, S. Stern et al., Femtosecond photoelectron diffraction on laser-aligned molecules: Towards time-resolved imaging of molecular structure, Phys. Rev. A 88, 061402(R) (2013).

[13] M. G. Pullen, B. Wolter, A.-T. Le, M. Baudisch, M. Hemmer, A. Senftleben, C. D. Schröter, J. Ullrich, R. Moshammer, C. D. Lin, and J. Biegert, Imaging an aligned polyatomic molecule with laser-induced electron diffraction, Nat. Commun. 6, 7262 (2015). 
[14] B. Wolter, M. G. Pullen, A.-T. Le, M. Baudisch, K. DoblhoffDier, A. Senftleben, M. Hemmer, C. D. Schröter, J. Ullrich, T. Pfeifer, R. Moshammer, S. Gräfe, O. Vendrell, C. D. Lin, and J. Biegert, Ultrafast electron diffraction imaging of bond breaking in di-ionized acetylene, Science 354, 308 (2016).

[15] G. Kastirke et al., Photoelectron Diffraction Imaging of a Molecular Breakup Using an X-Ray Free-Electron Laser, Phys. Rev. X 10, 021052 (2020).

[16] H. Sann, T. Havermeier, C. Müller, H.-K. Kim, F. Trinter, M. Waitz, J. Voigtsberger, F. Sturm, T. Bauer, R. Wallauer, D. Schneider, M. Weller, C. Goihl, J. Tross, K. Cole, J. Wu, M. S. Schöffler, H. Schmidt-Böcking, T. Jahnke, M. Simon, and R. Dörner, Imaging the Temporal Evolution of Molecular Orbitals during Ultrafast Dissociation, Phys. Rev. Lett. 117, 243002 (2016).

[17] Z. Vager, R. Naaman, and E. P. Kanter, Coulomb explosion imaging of small molecules, Science 244, 426 (1989).

[18] H. Stapelfeldt, E. Constant, H. Sakai, and P. B. Corkum, Timeresolved Coulomb explosion imaging: A method to measure structure and dynamics of molecular nuclear wave packets, Phys. Rev. A 58, 426 (1998).

[19] Th. Ergler, A. Rudenko, B. Feuerstein, K. Zrost, C. D. Schröter, R. Moshammer, and J. Ullrich, Spatiotemporal Imaging of Ultrafast Molecular Motion: Collapse and Revival of the $\mathrm{D}_{2}{ }^{+}$ Nuclear Wave Packet, Phys. Rev. Lett. 97, 193001 (2006).

[20] A. Hishikawa, A. Matsuda, M. Fushitani, and E. J. Takahashi, Visualizing Recurrently Migrating Hydrogen in Acetylene Dication by Intense Ultrashort Laser Pulses, Phys. Rev. Lett. 99, 258302 (2007).

[21] H. Ibrahim, B. Wales, S. Beaulieu, B. E. Schmidt, N. Thiré, E. P. Fowe, Éric Bisson, C. T. Hebeisen, V. Wanie, M. Giguére, J.-C. Kieffer, M. Spanner, A. D. Bandrauk, J. Sanderson, M. S. Schuurman, and F. Légaré, Tabletop imaging of structural evolutions in chemical reactions demonstrated for the acetylene cation, Nat. Commun. 5, 4422 (2014).

[22] M. Kunitski, S. Zeller, J. Voigtsberger, A. Kalinin, L. Ph. H. Schmidt, M. Schöffler, A. Czasch, W. Schöllkopf, R. E. Grisenti, T. Jahnke, D. Blume, and R. Dörner, Observation of the Efimov state of the helium trimer, Science 348, 551 (2015).

[23] C. E. Liekhus-Schmaltz, I. Tenney, T. Osipov, A. SanchezGonzalez, N. Berrah, R. Boll, C. Bomme, C. Bostedt, J. D. Bozek, S. Carron, R. Coffee, J. Devin, B. Erk, K. R. Ferguson, R. W. Field, L. Foucar, L. J. Frasinski, J. M. Glownia, M. Gühr, A. Kamalov et al., Ultrafast isomerization initiated by X-ray core ionization, Nat. Commun. 6, 8199 (2015).

[24] U. Ablikim, C. Bomme, H. Xiong, E. Savelyev, R. Obaid, B. Kaderiya, S. Augustin, K. Schnorr, I. Dumitriu, T. Osipov, R. Bilodeau, D. Kilcoyne, V. Kumarappan, A. Rudenko, N. Berrah, and D. Rolles, Identification of absolute geometries of cis and trans molecular isomers by Coulomb explosion imaging, Sci. Rep. 6, 38202 (2016).

[25] R. Guillemin, P. Decleva, M. Stener, C. Bomme, T. Marin, L. Journel, T. Marchenko, R. K. Kushawaha, K. Jänkälä, N. Trcera, K. P. Bowen, D. W. Lindle, M. N. Piancastelli, and M. Simon, Selecting core-hole localization or delocalization in $\mathrm{CS}_{2}$ by photofragmentation dynamics, Nat. Commun. 6, 6166 (2015).

[26] S. Pathak, R. Obaid, S. Bhattacharyya, J. Bürger, X. Li, J. Tross, T. Severt, B. Davis, R. C. Bilodeau, C. A. Trallero-Herrero,
A. Rudenko, N. Berrah, and D. Rolles, Differentiating and quantifying gas-phase conformational isomers using Coulomb explosion imaging, J. Phys. Chem. Lett. 11, 10205 (2020).

[27] L. Ph. H. Schmidt, T. Jahnke, A. Czasch, M. Schöffler, H Schmidt-Böcking, and R. Dörner, Spatial Imaging of the $\mathrm{H}_{2}{ }^{+}$ Vibrational Wave Function at the Quantum Limit, Phys. Rev. Lett. 108, 073202 (2012).

[28] S. Zeller, M. Kunitski, J. Voigtsberger, A. Kalinin, A. Schottelius, C. Schober, M. Waitz, H. Sann, A. Hartung, T. Bauer, M. Pitzer, F. Trinter, C. Goihl, C. Janke, M. Richter, G. Kastirke, M. Weller, A. Czasch, M. Kitzler, M. Braune et al., Imaging the $\mathrm{He}_{2}$ quantum halo state using a free electron laser, Proc. Natl. Acad. Sci. USA 113, 14651 (2016).

[29] T. Endo, S. P. Neville, V. Wanie, S. Beaulieu, C. Qu, J. Deschamps, P. Lassonde, B. E. Schmidt, H. Fujise, M. Fushitani, A. Hishikawa, P. L. Houston, J. M. Bowman, M. S. Schuurman, F. Légaré, and H. Ibrahim, Capturing roaming molecular fragments in real time, Science 370, 1072 (2020).

[30] M. Pitzer, M. Kunitski, A. S. Johnson, T. Jahnke, H. Sann, F. Sturm, L. Ph. H. Schmidt, H. Schmidt-Böcking, R. Dörner, J. Stohner, J. Kiedrowski, M. Reggelin, S. Marquardt, A. Schießer, R. Berger, and M. S. Schöffler, Direct determination of absolute molecular stereochemistry in gas phase by Coulomb explosion imaging, Science 341, 1096 (2013).

[31] B. Ulrich, A. Vredenborg, A. Malakzadeh, L. Ph. H. Schmidt, T. Havermeier, M. Meckel, K. Cole, M. Smolarski, Z. Chang, T. Jahnke, and R. Dörner, Imaging of the structure of the Argon and Neon dimer, trimer, and tetramer, J. Phys. Chem. A 115, 6936 (2011).

[32] B. Erk et al., Ultrafast Charge Rearrangement and Nuclear Dynamics upon Inner-Shell Multiple Ionization of Small Polyatomic Molecules, Phys. Rev. Lett. 110, 053003 (2013).

[33] B. Erk, R. Boll, S. Trippel, D. Anielski, L. Foucar, B. Rudek, S. W. Epp, R. Coffee, S. Carron, S. Schorb, K. R. Ferguson, M. Swiggers, J. D. Bozek, M. Simon, T. Marchenko, J. Küpper, I. Schlichting, J. Ullrich, C. Bostedt, D. Rolles et al., Imaging charge transfer in iodomethane upon x-ray photoabsorption, Science 345, 288 (2014).

[34] K. Motomura, E. Kukk, H. Fukuzawa, S.-i. Wada, K. Nagaya, S. Ohmura, S. Mondal, T. Tachibana, Y. Ito, R. Koga, T. Sakai, K. Matsunami, A. Rudenko, C. Nicolas, X.-J. Liu, C. Miron, Y. Zhang, Y. Jiang, J. Chen, M. Anand et al., Charge and nuclear dynamics induced by deep inner-shell multiphoton ionization of $\mathrm{CH}_{3} \mathrm{I}$ molecules by intense x-ray free-electron laser pulses, J. Phys. Chem. Lett. 6, 2944 (2015).

[35] A. Rudenko, L. Inhester, K. Hanasaki, X. Li, S. J. Robatjazi, B. Erk, R. Boll, K. Toyota, Y. Hao, O. Vendrell, C. Bomme, E. Savelyev, B. Rudek, L. Foucar, S. H. Southworth, C. S. Lehmann, B. Kraessig, T. Marchenko, M. Simon, K. Ueda et al., Femtosecond response of polyatomic molecules to ultra-intense hard x-rays, Nature (London) 546, 129 (2017).

[36] R. Dörner, V. Mergel, O. Jagutzki, L. Spielberger, J. Ullrich, R. Moshammer, and H. Schmidt-Böcking, Cold target recoil ion momentum spectroscopy: A momentum microscope to view atomic collision dynamics, Phys. Rep. 330, 95 (2000).

[37] J. Ullrich, R. Moshammer, A. Dorn, R. Dörner, L. Ph. H. Schmidt, and H. Schmidt-Böcking, Recoil-ion and electron momentum spectroscopy: Reaction-microscopes, Rep. Prog. Phys. 66, 1463 (2003). 
[38] W. Decking et al., A MHz-repetition-rate hard x-ray freeelectron laser driven by a superconducting linear accelerator, Nat. Photonics 14, 391 (2020).

[39] S. Lloyd, Least squares quantization in PCM, IEEE Trans. Inf. Theory 28, 129 (1982).

[40] J. MacQueen, Some methods for classification and analysis of multivariate observations, in Proceedings of the Fifth Berkeley Symposium on Mathematical Statistics and Probability (University of California Press, Berkeley, CA, USA, 1967), Vol. 1, pp. 281-297.

[41] F. Pedregosa, G. Varoquaux, A. Gramfort, V. Michel, B. Thirion, O. Grisel, M. Blondel, P. Prettenhofer, R. Weiss, V. Dubourg, J. Vanderplas, A. Passos, D. Cournapeau, M. Brucher, M. Perrot, and É. Duchesnay, Scikit-learn: Machine learning in Python, J. Mach. Learn. Res. 12, 2825 (2011).

[42] NIST Standard Reference Database 101, http://cccbdb.nist. gov/.

[43] X. Li, L. Inhester, S. Robatjazi, B. Erk, R. Boll, K. Hanasaki, K. Toyota, Y. Hao, C. Bomme, B. Rudek, L. Foucar, S. Southworth, C. Lehmann, B. Kraessig, T. Marchenko, M. Simon, K. Ueda, K. Ferguson, M. Bucher, T. Gorkhover et al., Pulse Energy and Pulse Duration Effects in the Ionization and Fragmentation of Iodomethane by Ultraintense Hard X Rays, Phys. Rev. Lett. 127, 093202 (2021).

[44] X. Li, R. Boll, D. Rolles, and A. Rudenko, Simple model for sequential multiphoton ionization by ultraintense $\mathrm{x}$ rays, Phys. Rev. A 104, 033115 (2021).

[45] Y. Hao, L. Inhester, K. Hanasaki, S.-K. Son, and R. Santra, Efficient electronic structure calculation for molecular ionization dynamics at high x-ray intensity, Struct. Dyn. 2, 041707 (2015).

[46] L. Inhester, K. Hanasaki, Y. Hao, S.-K. Son, and R. Santra, $\mathrm{X}$-ray multiphoton ionization dynamics of a water molecule irradiated by an x-ray free-electron laser pulse, Phys. Rev. A 94, 023422 (2016).

[47] P. Virtanen, R. Gommers, T. E. Oliphant, M. Haberland, T. Reddy, D. Cournapeau, E. Burovski, P. Peterson, W. Weckesser, J. Bright, S. J. van der Walt, M. Brett, J. Wilson, K. J. Millman,
N. Mayorov, A. R. J. Nelson, E. Jones, R. Kern, E. Larson, C. J. Carey et al., SciPy 1.0: Fundamental algorithms for scientific computing in Python, Nat. Methods 17, 261 (2020).

[48] R. Storn and K. Price, Differential evolution-A simple and efficient heuristic for global optimization over continuous spaces, J. Global Optim. 11, 341 (1997).

[49] Z. Wei, J. Li, L. Wang, S. T. See, M. H. Jhon, Y. Zhang, F. Shi, M. Yang, and Z.-H. Loh, Elucidating the origins of multimode vibrational coherences of polyatomic molecules induced by intense laser fields, Nat. Commun. 8, 735 (2017).

[50] Y. Malakar, W. L. Pearson, M. Zohrabi, B. Kaderiya, K. R. P, F. Ziaee, S. Xue, A. T. Le, I. Ben-Itzhak, D. Rolles, and A. Rudenko, Time-resolved imaging of bound and dissociating nuclear wave packets in strong-field ionized iodomethane, Phys. Chem. Chem. Phys. 21, 14090 (2019).

[51] https://doi.org/10.22003/xfel.eu-data-002159-00.

[52] M. Lundqvist, D. Edvardsson, P. Baltzer, and B. Wannberg, Doppler-free kinetic energy release spectrum of $\mathrm{N}_{2}^{2+}$ J. Phys. B: At., Mol. Opt. Phys. 29, 1489 (1996).

[53] Th. Weber, O. Jagutzki, M. Hattass, A. Staudte, A. Nauert, L. Schmidt, M. H. Prior, A. L. Landers, A. Bräuning-Demian, H. Bräuning, C. L. Cocke, T. Osipov, I. Ali, R. Díez Muiño, D. Rolles, F. J. García de Abajo, C. S. Fadley, M. A. Van Hove, A. Cassimi, H. Schmidt-Böcking et al., K-shell photoionization of $\mathrm{CO}$ and $\mathrm{N}_{2}$ : Is there a link between the photoelectron angular distribution and the molecular decay dynamics? J. Phys. B: At., Mol. Opt. Phys. 34, 3669 (2001).

[54] K. Fehre, D. Trojanowskaja, J. Gatzke, M. Kunitski, F. Trinter, S. Zeller, L. Ph. H. Schmidt, J. Stohner, R. Berger, A. Czasch, O. Jagutzki, T. Jahnke, R. Dörner, and M. S. Schöffler, Absolute ion detection efficiencies of microchannel plates and funnel microchannel plates for multi-coincidence detection, Rev. Sci. Instrum. 89, 045112 (2018).

[55] P. J. Rousseeuw and K. V. Driessen, A fast algorithm for the minimum covariance determinant estimator, Technometrics $\mathbf{4 1}$, 212 (1998). 